\title{
Alignment-free methods for metagenomic profiling
}

\author{
Shanshan Gao, Diem-Trang Pham, Vinhthuy Phan \\ From 14th Annual UT-KBRIN Bioinformatics Summit 2015 \\ Buchanan, TN, USA. 20-22 March 2015
}

\section{Background}

The primary goal of metagenomic studies is to analyze and evaluate the rich microbial communities present in all natural environments. The construction and utilization of a large index required by alignment-based methods for thousands of microbial genomes can be computationally prohibitive. To avoid this computational cost, we investigated three different variations of an alignmentfree method for profiling abundances of microbial communities.

\section{Materials and methods}

The main idea of the method is reformulate the problem of determining abundance of microbial genomes as finding optimal solutions of linear equations that satisfy specific constraints. A set of genomic markers for the entire set of genomes is represented by a matrix $F$, where $\mathbf{F}_{\mathbf{i j}}$ represents the frequency of marker $\mathbf{i}$ in genome $\mathbf{j}$. The occurrence vector $\mathbf{b}$ represents the frequencies of markers in reads. We would like to find an optimal solution $\mathbf{x}$, the abundance vector in which $\mathbf{x}_{\mathbf{j}}$ represents the abundance of genome $\mathbf{j}$. To find the abundance vector $\mathrm{x}$, we solve the linear equation $\mathbf{F x}=\mathbf{b}$. The methods to choose $\mathrm{F}$ and $\mathrm{b}$ are the key factor to find the optimal value of $\mathrm{x}$. We introduced a concept of genome specific marker (GSM), which is a kmer that occurs in only one genome and no other. We exhaustively determine such markers from the entire dataset and represent the frequencies of these markers in the matrix F. Given a set of reads from a metagenomic dataset, we compute the frequency of GSM as b. Then, three variations can be formulated, respectively, as a linear programming problem (LP), a least-square approximation problem (L2), and an L1-approximation problem.

\footnotetext{
* Correspondence: vphan@memphis.edu

Department of Computer Science, University of Memphis, Memphis, TN
} 38152, USA and take full advantage of:

- Convenient online submission

- Thorough peer review

- No space constraints or color figure charges

- Immediate publication on acceptance

- Inclusion in PubMed, CAS, Scopus and Google Scholar

- Research which is freely available for redistribution 